Research Article

\title{
Knowledge and Attitudes Regarding Seasonal Influenza and Influenza Vaccination among Patients and Their Companions in North Palestine Hospitals
}

\author{
Issa Alawneh $\mathbb{D}^{1},{ }^{1}$ Hamza Al-Sayeh, ${ }^{1}$ Mahdi Zaid, ${ }^{1}$ Maysa Alawneh, ${ }^{1}$ and Hossam Al-Tatari ${ }^{2}$ \\ ${ }^{1}$ Department of Pediatrics, An-Najah National University Hospital, Nablus, State of Palestine \\ ${ }^{2}$ The Heart Medical Center, Al Ain, UAE
}

Correspondence should be addressed to Issa Alawneh; issaalawneh2007@hotmail.com

Received 12 April 2021; Accepted 11 September 2021; Published 15 September 2021

Academic Editor: Carol J. Burns

Copyright (c) 2021 Issa Alawneh et al. This is an open access article distributed under the Creative Commons Attribution License, which permits unrestricted use, distribution, and reproduction in any medium, provided the original work is properly cited.

Background. Seasonal influenza is a common highly infectious disease that can affect the upper and lower airway in children and adults mainly in wintertime which is caused by many different influenza viruses and, in some cases, may cause serious complications such as pneumonia. We conducted this study to assess the knowledge and attitudes among patients and their companions in North Palestine hospitals regarding seasonal influenza and influenza vaccination and factors influencing the uptake of this vaccine so that we can identify gaps in their knowledge and give feedback to health authorities for future quality improvement projects by increasing awareness of its effectiveness and safety. Methods. A cross-sectional 17-item survey included randomly selected samples of 327 North Palestinian patients and their companions at North Palestine hospitals. Result. A total of 327 completed questionnaires were received with a response rate of $92 \%$. Of these, 129 participants (39.4\%) believed that influenza is the same as common cold. The majority of participants (85.3\%) had heard of the influenza vaccine before. Although nearly half of them $(53.6 \%)$ believed that the influenza vaccine is safe, only $112(34.7 \%)$ of the participants considered vaccination an effective means in preventing serious influenza-related complications and only $89(27.2 \%)$ participants were previously vaccinated. The main reasons for not being vaccinated included that vaccination is not necessary because flu is not a serious disease (67\%), concerns about vaccine efficacy and its side effects (25.6\%), fear of needles and injection (25.2\%), and $17.8 \%$ of the participants believed that this vaccine is expensive. Conclusion. The uptake and knowledge of the influenza vaccine among Palestinians are low. Vaccinated participants in our survey showed a higher level of knowledge compared to nonvaccinated participants. Half of the participants believed in the safety of the vaccine and one-third of them believed in its efficacy in preventing flu illness and its complications. Extensive and sustained efforts are needed by public health programs to promote the flu vaccine among the public by increasing awareness of its effectiveness and safety.

\section{Introduction}

Seasonal influenza is a common highly infectious disease that can affect the upper and lower airway in children and adults, mainly in wintertime, which is caused by many different influenza viruses and, in some cases, may cause serious complications such as pneumonia. Yearly, between 250000 and 500000 deaths of the world's population are estimated due to influenza virus [1]. It is known that influenza virus has high mutation rates, and it has been shown that its annual epidemics is $5-15 \%$ of the global population, causing 290,000-650,000 deaths worldwide annually [2, 3]. Moreover, influenza is also associated with a significant economic burden on healthcare costs [4].

Influenza vaccination is a safe and effective primary available tool for the prevention of influenza and reduction of economic and social burden. Indeed, seasonal influenza vaccination is the most effective strategy for preventing influenza virus infection and its complications [5]. However, despite the national recommendations that encourage this vaccination taken, the rates of vaccination uptake remain low $[6,7]$. 
Numerous national studies have proved the effectiveness of annual influenza vaccination in the reduction of mortality and morbidity, especially in high-risk groups such as the elderly, chronically ill patients, pregnant, and children $[8,9]$. For example, in the USA, during the season of influenza between 2017 and 2018, vaccination prevented approximately 7 million influenza illnesses, 109,000 hospitalizations, and 8,000 deaths [10].

The varying influenza vaccine coverage across countries corresponds to the level of knowledge and attitude toward seasonal influenza vaccines, both in the general population and in the at-risk groups: individuals with little knowledge and a negative attitude towards vaccination are usually not vaccinated [11-14].

Previous studies showed that the influenza vaccine was usually significantly protective and that most clinicians and family physicians were supportive of vaccination and had good knowledge of vaccination indications [15-19].

In Palestine, infectious diseases cause less than $10 \%$ of all deaths; respiratory diseases (ICD10 code: J00-J99.9) cause $70 \%$ of those deaths with a mortality rate of 17.0 per 100,000 population during 2016, being the sixth most common cause of death [20].

Overall, influenza viruses were the most common cause of respiratory tract infections (RTIs) among hospitalized Palestinian patients in the West Bank. Children and the elderly were the most affected with RTIs. The elderly population ( $\geq 60$ years old) had the highest rates [21].

In Palestine, the influenza vaccine is not included in the Palestinian National Immunization program and not covered by insurance except for healthcare workers.

No previously published studies are focused on receiving influenza vaccine and assessing Palestinian population knowledge about vaccine-related risks and vaccine safety.

In this study, we conducted a cross-sectional study on a sample of the Palestinian population in North Palestine hospitals to assess public knowledge, attitudes, and practices (KAP) related to influenza illness and influenza vaccination.

\section{Methods}

2.1. Study Design and Setting. A descriptive cross-sectional survey was conducted during a period of 3 months from June to August 2018 of patients and their companions at North Palestine hospitals, both in the private and governmental sectors, involving five major cities in North Palestine including Nablus, Jenin, Tulkarm, Tubas, and Qalqilia.

Inclusions criteria: male or female, at least 18 years of age, married, and at least have one dependent child

Exclusion criteria: less than 18 years of age, single, and married but does not have children

2.2. The Questionnaire and Data Collection. To achieve the aim of our study, we used a structured 17-item questionnaire assessing the knowledge and attitude towards influenza illness and its vaccination consisting of three main parts.
TABLE1: Demographic characters of the study sample $(n=327)$.

\begin{tabular}{lc}
\hline Demographics & Percentages (\%) \\
\hline Gender & 56 \\
Male & 44 \\
Female & \\
\hline Age $(y r)$ & 51.1 \\
$20-30$ & 26.6 \\
$31-40$ & 12.2 \\
$41-50$ & 10.1 \\
$>50$ & \\
\hline Level of education & 4.3 \\
Primary & 22.3 \\
Secondary & 73.4 \\
University and higher &
\end{tabular}

(1) Demographic information: sex, age, and education level

(2) General knowledge about influenza illness (five questions)

(3) Knowledge and attitude toward the influenza vaccine (ten questions)

We modified a questionnaire used in Pretoria, South Africa, assessing knowledge, attitudes, and practices regarding seasonal influenza and influenza vaccination among diabetics in September 2015 [22]. It was reviewed and approved by the An-Najah National University Institution Review Board (IRB). Then, the permissions to conduct the research at the Palestinian $\mathrm{MOH}$-related facilities were obtained from Palestinian $\mathrm{MOH}$ as well as from the private sectors visited prior to the study start date.

Written informed consent was taken from all participants before they completed the questionnaire. We used a convenient sampling method in this study.

All collected data were treated with confidentiality and would be used for research purposes only.

2.3. Statistical Analysis. Answers of the population responded were translated into an Excel sheet and then whether the respondents strongly agreed or just agree with a good practice; the answers were considered true, as if he strongly disagrees or just disagree with a wrong practice.

We considered any question with returned correct answers of less than $90 \%$ as a "Gap" in participant knowledge.

\section{Results}

A total number of 327 patients and their companions participated in the study with a response rate of 92.0\% (301/ $327)$. Most of the participants $(167 / 327,51.1 \%)$ were between 20-30 years; out of them, 56\% were females (Table 1).

When asked about influenza, the majority believed that flu was caused by the virus $(289 / 327,88.4 \%)$, while 129 participants $(39.4 \%)$ still believed that it is the same as common cold.

Regarding the major symptoms of influenza, the most frequent choices were muscle ache (263/327, 80.4\%), fever 
TABLE 2: Surveyed questions and responses to each question.

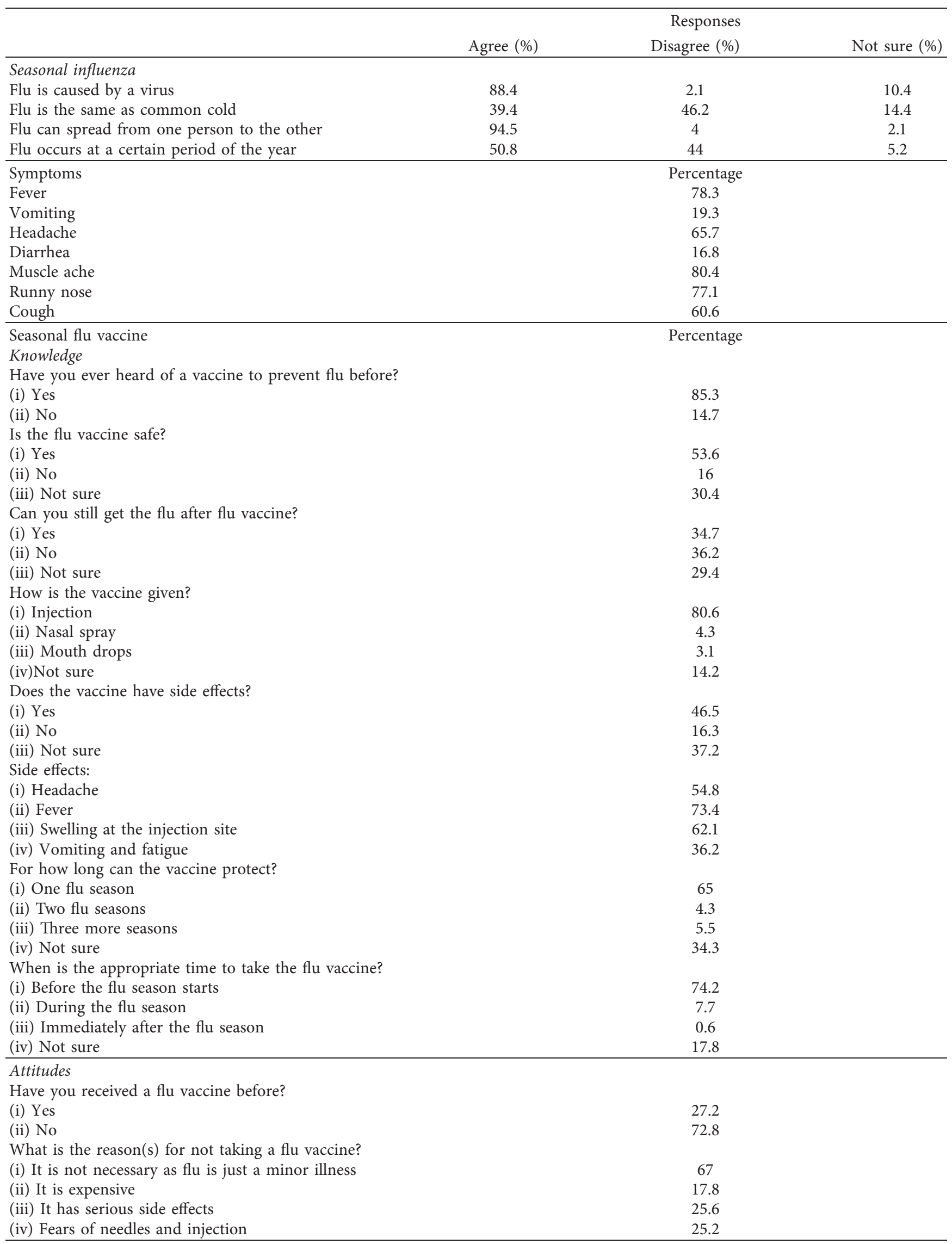


TABLe 3: Seasonal flu knowledge scores of participants $(n=327)$.

\begin{tabular}{lc}
\hline Seasonal flu knowledge score & Participants (\%) \\
\hline Good seasonal flu knowledge & 69.9 \\
Poor seasonal flu knowledge & 30.1 \\
\hline
\end{tabular}

TABLE 4: Vaccine knowledge scores of participants $(n=327)$.

\begin{tabular}{lc}
\hline Flu vaccine knowledge score & Participants (\%) \\
\hline Good vaccine knowledge & 53.4 \\
Poor vaccine knowledge & 46.6 \\
\hline
\end{tabular}

$(256 / 327,78.3 \%)$, runny nose $(252 / 327,77.1 \%)$, headache (215/327, 65.7\%), and cough (198/327, 60.6\%) (Table 2).

Out of the $85.3 \%(279 / 327)$ of participants who had heard of the influenza vaccine previously to prevent flu, only $171 / 327,53.6 \%$, indicated that the vaccine is safe and $83.8 \%$ (140/167), although $36.2 \%$ (117/327) of the participants indicated that one can still develop flu despite being vaccinated.

Nearly half of the participants $(151 / 327,46.5 \%)$ reported known side effects of the vaccine, including fever $(73.4 \%)$, headache $(54.8 \%)$, swelling at the injection site $(62.1 \%)$, and vomiting with fatigue (36.2\%). Nearly half of the participants $(183 / 327,56 \%)$ indicated that it can only protect for one flu season and that $74.2 \%$ of them know that the appropriate time to take the vaccine is before the flu season starts (242/ 327). About $69.9 \%$ and $53.4 \%$ had good knowledge about seasonal flu and vaccine, respectively (Tables 3 and 4).

When asked about their previous influenza vaccination practice, only $27.2 \%(89 / 327)$ had previously been vaccinated at one point in the time of their lives. A majority of the participants had never received influenza vaccination before $(238 / 327,72.8 \%)$, and the main reasons given by them included that vaccination is not necessary because flu is just a minor illness $(67 \%), \%)$, concerns about vaccine efficacy and its side effects (25.6\%), fear of needles and injection (25.2\%), and $17.8 \%$ of the participants believed that this vaccine is expensive.

\section{Discussion}

This study is the first of its kind in Palestine to investigate and assess the knowledge and attitudes of populations regarding influenza illness and its vaccines.

In this study, although approximately half of participants had good knowledge scores about seasonal flu and its vaccine, only one-third of them had taken the influenza vaccine previously and nearly half of them believed that the vaccine may have many side effects.

In keeping with that, the average cost of influenza vaccine in Palestine is near 13 US dollars, and the cost is not covered by health insurance. These significant fears of side effects, false belief about vaccine safety, limited availability and coverage of vaccination services, and even poor knowledge of the vaccine's benefits ultimately led to lower vaccination rates among the general population in North Palestine. These findings highlight the need to increase efforts and awareness in the community regarding influenza illness and vaccination to reduce the economic and social burden.

The utilitarian value of vaccination in enhancing public health to assure the population of the efficacy and safety of the vaccine by using media such as newspapers, posters, brochures, and mobile messages with adequate education received from medical staff proved to be the most effective tools for improving influenza vaccination practices in North Palestine and can greatly simplify this process [23]. Moreover, many studies advised that mandatory freely available vaccination could be helpful in limiting disease burden $[24,25]$.

\section{Conclusions}

Overall, the uptake and knowledge of the influenza vaccine among Palestinians are low. Vaccinated participants in our survey showed a higher level of knowledge compared to nonvaccinated participants. Half of the participants believed in the safety of the vaccine, and one-third of them believed in its efficacy in preventing flu illness as well as its known complications. Extensive and sustained efforts are needed by public health programs to promote the flu vaccine among the public by increasing awareness of its effectiveness and safety.

5.1. Limitations. This study has some limitations. Specifically, the study occurred in North Palestine hospitals and may not be generalizable to other regions of Palestine. Additionally, the study sample size was small which was drawn from a selected population in hospitals, so a significant selection bias cannot, therefore, be ruled out.

\section{Data Availability}

The datasets generated and analysed during the current study are not publicly available due to participant private polices and research regulation agreement related to Najah National University but are available from the corresponding author on reasonable request.

\section{Ethical Approval}

The authors confirm that all methods were carried out in accordance with relevant guidelines and regulations related to Najah National University and the Palestinian Ministry of Health. The study was approved by the An-Najah National University Institution Review Board (IRB).

\section{Consent}

Written informed consent was obtained from all subjects or/ and, if subjects are under 18, from a parent and/or legal guardian.

\section{Conflicts of Interest}

None of the authors declares conflicts of interest. 


\section{Authors' Contributions}

Issa Alawneh prepared the proposal and wrote and edited the manuscript. Hamza Al-Sayeh helped in data collection. Mahdi Zaid divided the work among the investigators, collected data, and conducted analysis in addition to preparing the tables. Maysa Alawneh as the first supervisor reviewed the data analysis and the manuscript and guided steps to conduct the research step by step. Hossam Al-Tatari as the second supervisor helped in the idea of the research, emphasized the importance of the study, and helped in validating the questioner, as well as guided the research flow and monitored the steps from the proposal to data entry.

\section{References}

[1] World Health Organization, A Manual for Estimating Disease Burden Associated with Seasonal Influenza, World Health Organization, Geneva, Switzerland, 2015.

[2] World Health Organization, Influenza (Seasonal), World Health Organization, Geneva, Switzerland, 2018.

[3] A. D. Iuliano, K. M. Roguski, H. H. Chang et al., "Estimates of global seasonal influenza-associated respiratory mortality: a modelling study," Lancet (London, England), vol. 391, no. 10127, pp. 1285-1300, 2018.

[4] P. R. Blank and T. D. Szucs, "Increasing influenza vaccination coverage in recommended population groups in Europe," Expert Review of Vaccines, vol. 8, no. 4, pp. 425-433, 2009.

[5] L. A. Grohskopf, E. Alyanak, K. R. Broder, E. B. Walter, A. M. Fry, and D. B. Jernigan, "Prevention and control of seasonal influenza with vaccines: recommendations of the advisory committee on immunization practices-United States, 2019-20 influenza season," MMWR. Recommendations and Reports, vol. 68, no. 3, pp. 1-21, 2019.

[6] H. G. Hollmeyer, F. Hayden, G. Poland, and U. Buchholz, "Influenza vaccination of health care workers in hospitals-a review of studies on attitudes and predictors," Vaccine, vol. 27, no. 30, pp. 3935-3944, 2009.

[7] O. Yaqub, S. Castle-Clarke, N. Sevdalis, and J. Chataway, "Attitudes to vaccination: a critical review," Social Science \& Medicine, vol. 112, pp. 1-11, 2014.

[8] A. Pless, S. R. McLennan, D. Nicca, D. M. Shaw, and B. S. Elger, "Reasons why nurses decline influenza vaccination: a qualitative study," BMC Nursing, vol. 16, no. 1, p. 20, 2017.

[9] D. J. Opel, J. Heritage, J. A. Taylor et al., “The architecture of provider-parent vaccine discussions at health supervision visits," Pediatrics, vol. 132, no. 6, pp. 1037-1046, 2013.

[10] Centers for Disease Control and Prevention (CDC), 20162017 Estimated Influenza Illnesses, Medical Visits, and Hospitalizations Averted by Vaccination in the United States, CDC, Atlanta, Georgia, 2018.

[11] M. P. S. Yeung, F. L. Y. Lam, and R. Coker, "Factors associated with the uptake of seasonal influenza vaccination in adults: a systematic review," Journal of Public Health, vol. 38, Article ID fdv194, 2016.

[12] A. Kamimura, H. N. Trinh, S. Weaver et al., "Knowledge and perceptions of influenza vaccinations among college students in Vietnam and the United States," Journal of Preventive Medicine and Public Health, vol. 50, no. 4, pp. 268-273, 2017.

[13] G. El Khoury and P. Salameh, "Influenza vaccination: a crosssectional survey of knowledge, attitude and practices among the lebanese adult population," International Journal of
Environmental Research and Public Health, vol. 12, no. 12, pp. 15486-15497, 2015.

[14] Y. Ma, T. Li, W. Chen, J. Chen, M. Li, and Z. Yang, "Knowledge, attitudes and practices (KAP) toward seasonal influenza vaccine among young workers in south China," Human Vaccines \& Immunotherapeutics, vol. 14, no. 5, pp. 1283-1293, 2018.

[15] Y.-C. Chang, Y.-J. Chou, J.-Y. Liu, T.-F. Yeh, and N. Huang, "Additive benefits of pneumococcal and influenza vaccines among elderly persons aged 75 years or older in Taiwan-a representative population-based comparative study," Journal of Infection, vol. 65, no. 3, pp. 231-238, 2012.

[16] E. Dube, V. Gilca, C. Sauvageau et al., "Canadian family physicians' and paediatricians' knowledge, attitudes and practices regarding $\mathrm{A}(\mathrm{H} 1 \mathrm{~N} 1)$ pandemic vaccine," BMC Research Notes, vol. 3, p. 102, 2010.

[17] J. M. McAnerney, S. Walaza, A. L. Cohen et al., "Effectiveness and knowledge, attitudes and practices of seasonal influenza vaccine in primary healthcare settings in south Africa, 2010-2013," Influenza and Other Respiratory Viruses, vol. 9, no. 3, pp. 143-150, 2015.

[18] M. T. Osterholm, N. S. Kelley, A. Sommer, and E. A. Belongia, "Efficacy and effectiveness of influenza vaccines: a systematic review and meta-analysis," The Lancet Infectious Diseases, vol. 12, no. 1, pp. 36-44, 2012.

[19] J. K. Breteler, J. S. Tam, M. Jit, J. C. F. Ket, and M. R. De Boer, "Efficacy and effectiveness of seasonal and pandemic A (H1N1) 2009 influenza vaccines in low and middle income countries: a systematic review and meta-analysis," Vaccine, vol. 31, no. 45, pp. 5168-5177, 2013.

[20] Ministry-of-Health, Health Annual Report: Palestine, Ministry-of-Health, Ramallah, Palestine, 2016.

[21] R. Abu Seir, W. Njoum, R. Najajrah et al., "Acute respiratory tract infections among hospitalized palestinian patients (2011-2016): a retrospective study," The Canadian Journal of Infectious Diseases \& Medical Microbiology, vol. 2021, Article ID 5643134, 8 pages, 2021.

[22] O. D. Olatunbosun, T. M. Esterhuizen, and C. S. Wiysonge, "A cross sectional survey to evaluate knowledge, attitudes and practices regarding seasonal influenza and influenza vaccination among diabetics in Pretoria, south Africa," Vaccine, vol. 35, no. 47, pp. 6375-6386, 2017.

[23] M. M. Masadeh, K. H. Alzoubi, S. I. Al-Azzam, H. S. Al-Agedi, B. E. Abu Rashid, and T. L. Mukattash, "Public awareness regarding children vaccination in Jordan," Human Vaccines \& Immunotherapeutics, vol. 10, no. 6, pp. 1762-1766, 2014, p.

[24] A. L. Ottenberg, J. T. Wu, G. A. Poland, R. M. Jacobson, B. A. Koenig, and J. C. Tilburt, "Vaccinating health care workers against influenza: the ethical and legal rationale for a mandate," American Journal of Public Health, vol. 101, no. 2, pp. 212-216, 2011, p.

[25] J. J. M. van Delden, R. Ashcroft, A. Dawson, G. Marckmann, R. Upshur, and M. F. Verweij, "The ethics of mandatory vaccination against influenza for health care workers," Vaccine, vol. 26, no. 44, pp. 5562-5566, 2008, p. 